Trieste

\title{
SPIGOLATURE TOPONOMASTICHE CARSICHE
}

\section{Monte coste}

Denominazione di cui si ignora 1'"origine", annota Carlo Chersi nella sua accurata guida Itinerari del Carso triestino (giunta postuma alla sua settima edizione, Trieste 1984), a proposito di coste, nome di un colle abbastanza alto (m. 410) che si eleva alle spalle di Sales (Salež), nella catena cui appartengono anche il Monte San Leonardo (Sv. Leonard), a. N. O., e la Vetta Grande (Ostri Vrh) a S. E., ed è chiamato oggi localmente Gradec (in quanto sede di castelliere, cfr. A. M. Radmilli ASPP 2, 193-75, p. 126 e già C. Marchesetti Castellieri, Trieste 1903, p. 37) o anche (Monte) Dernovesch (Dernovesh), grafie tedeschizzanti di uno slov. (dial.) Dernovcah (dall'agg. drnovec "provvisto di zolle erbose"). L'etimo di questo coste è, invece, assai ovvio, non appena si ponga mente a poche cose. Primo, coste (anche Costa, Tavoletta dell'Istituto Geogr. Militare al 50.000 ) non è che un'italianizzazione recente (la troviamo attestata a partire dal 1949) di un più antico Kosten, che ricorre, afferma il Chersi l.c., nelle vecchie carte austriache, ossia anteriormente al 1914 (ne ho trovato un esempio nella Guida dei Dintorni di Trieste, p. 96, che data del 1909), e che viene impiegato tuttora in alternanza col costa e coste citati. Secondo, a differenza di costa (o coste) il nome kosten ha un suo significato, ben netto e preciso, e benche risulti denominazione ormai desueta presso gli sloveni del carso (che preferiscono, come s'è detto, le denominazioni più moderne Gradec, o Gradc, e Dernovcah, v. Tržaško ozemlje D 2 e D. Cannarella presso Marchesetti Castellieri, ristampa 1981, p. 25), non v'ha dubbio che esso vada ricondotto alla voce slovena che significa "castagno", precisamente a kóstanj (da lat. CASTANEUM, v. F.Bezlaj, ESSJ II, 1982, p. 71), di cui kosten rappresenta una vecchia forma dialettale, qualcosa come kostënj, con ë trascritto e e con depalatizzazione della nasale palatale finale. Denominazione riferita quindi al manto vege- 
tale più antico che contraddistingueva questo monte (nei dintorni di sales il castagno, tuttora, non è un albero tanto raro), cui si oppone, nettamente, quella di Dernovcah, nata più tarai col mutare del paesaggio circostante (o del tipo di osservazione nei suoi riguardi).

\section{Percedol}

Anche per la soluzione del problema dell'etimo di percedol (grafia slov. Perčedol, dial. Prčji dol, Tržaško ozemlje F 4), denominazione della nota conca con laghetto lungo la strada opicina (opčina) - Monrupino (Repentabor) sono decisive le testimonianze più antiche, questa volta risalenti al XVII sec. In uno schizzo topografico risalente all'a. 1643 c. (Arch. diplom. Triest. 12 A $2 / 16$ IV) troviamo infatti l'importante forma pertidol, la quale si ripete in un altro documento coevo (Atto di confinazione, Arch. Diplom. cit. $\beta$ A 14 [bis]), nonche in un documento del 1645 (Arch. Diplom. cit. A 14: "nel locho sopra memmorato di Pertidol" e, più avanti," caua (=cavità, dolina) grande detta Pertidol, si trova un'aqua ecc."). Forma di passaggio rispetto ai più moderni percedu (Plan V. Triest, 1843) e Percedol (Guida dei dintorni di Trieste, Trieste 1909, p. 69 e

tav. p. 129) e senza dubbio perichian-Dol, dell'a. 1701 (P. Kandler, Confinazioni p. 24, in "Miscellanea Conti"): "croce detta Perichian-Dol". Evidentemente abbiamo a che fare con un nome significante "valle putrida", con riferimento al laghetto o stagno che occupa il fondo della dolina. La prima parte del toponimo non è altro che l'aggettivo slov. perec (dal verbo pereti "imputridire, marcire"), il quale, nella fase più antica comportava una $t$ non ancora palatalizzata, qualcosa come *peretji; il ch(i) della forma citata dal Kandler potrebbe alludere ad una prima fase di palatalizzazione, peret' (o perek'), prima che si giunga a quella conclusiva, perec. Naturalmente, come succede assai spesso nei dialetti sloveni carsici, l'e mediano atono cade, e questo è un fatto compiuto (o in via di compimento) già nella forma più antica del toponimo, che comporta un perti-, non pereti-. Però, per quel periodo, la forma non sincopata del tipo pereti-, pereci- non era ancora scomparsa 
del tutto, dal momento che nel 1701 è attestato ancora perichi. Un problema a sé costituiscono le forme tipo Mercedou e Mercendul, attestate nelle Perticazioni degli a. 1647-1649 (estratto di de Jenner), quindi pressoche coeve al Pertidol ora esaminato. Se perti- non era ancora passato a perči-, è assai improbabile che cotesto Mercedol costituisca un'evoluzione a partire da percedol. D'altro canto esso non è una "ghost form", in quanto sopravvissuto fino ad oggi, a dar retta a M. Magajna Trieste in bianco e nero (Udine-Trieste 1983), il quale nella didascalia alla fot. 94 scrive, letteralmente, Mercedol. Essendo per questo Mercedol improponibile, ancine, un riavvicinamento a slov. mrzel (e corradicali) "freddo" ("conca fredda", con riferimento al fenomeno dell'inversione termica, riscontrabile quivi peró in modo assai poco rilevante, causa la scarsa profondità delia dolina), preferirei vedere in cotesto Mercedol una denominazione staccata, precisamente slov. mrc "nebbia secca, nebbione" ("der Höhenrauch", Wolf-Pleteršnik s.v.), con riferimento allo strato di nebbia che si forma durante la stagione invernale sulla superficie del laghetto, denominazione fusasi con quella di percedol, una volta che per(e)ti- passò a perči-, e considerata da allora come semplice variante fonetica della forma toponimica d'uso più diffuso. Una prova che anche percisia autonomo rispetta a Merci- la deduciamo del fatto che nelle. vicinanze di pertidol $i$ documenti secenteschi che abbiamo sopra citato attestano una localita assai prossima chiamata pertiduch (ossia perči duh "odore, esalazione di marcio"); come a dire che perti-era in quei paraggi come di casa, e che si era formata sul posto una piccola costellazione di toponimi con primo elemento perti-"marcio". Naturalmente, al limite, è anche possibile che esistesse originariamente solo mercedol e che pertidol. sia secondario, nato cioè per influsso del vicino pertiduh, fa difficoltà però il fatto che per una sostituzione di Merce-con Perce- era necessario che il -t(i)-fosse già palatalizzato, cosa che non è attestabile per un periodo così antico. Conviene quindi senz'altro ritenere la forma tipo pertidol, percidol del tutto originaria e autentica.

Un ultimo particolare: l'amico cl. Noliari riferisce che gli 
Sloveni della zona interpretano oggi Percedol come per če dol "verso (presso) quella valle". Alla luce delle considerazioni sopra esposte mi sembra che cotesta spiegazione valga unicamente come "etimo popolare", ossia paretimologia.

\section{Per Ciastel}

Nel mio articolo Alla ricerca di tracce di friulanità nella toponomastica del Carso triestino (SLF I, 1969, pp. 223-256) avevo dimostrato come la neolatinità, inserita in un contesto più compattamente sloveno, del Carso triestino e zone contermini fosse, là dove risultava attestata, di tipo spiccatamente friulano, assai simile alla friulanità triestina (tergestino). E adducevo a comprova di ciò toponimi come Brajda (cfr. friul. bxaida "podere in aperta campagna"), Brajca (da friulo bearz "resedio, podere recintato nei pressi di un centro abitato"), Fernetti (da friulaneggiante farnet "boco di farnie"), stellei (a. 1346) HASTELLĒTUM "bosco ceduo", Uersine (a. 1498), "(altu-ra) a forma di vomero" /cfr. friul. uarzine a vuarzine, ant. anche guarzine, "aratro", < lat. ORGINUM "strumento (da lavoro)"/ ecc. Ne mancava fra di essi qualche caso attestante con certezza il fenomeno, così caratteristico del friulano a partire da una certa epoca, di lat. CA passato all'iniziale di parola a chia, cja e menzionavo un chia (nei pressi di Santa Croce [Križ]), lett. "casa" (attestazioni parallele anche ca, cha). $L^{\prime}$ apporto di materiale più fresco mi offre ora la possibilità di convalidare ulteriormente codesto particolare aspetto della toponomastica carsica. Sul materiale ricavabile dal prezioso (e indispensabile) Tržaško ozemlje riferirò in altra sede. Qui mi basterà citare, riferendomi ad altra fonte, ugualmente insospettabile, Benedetto Lonza (ASPP 1, 1970-72), p. 48, ib. 2, 1973-74, p. 48), un bellissmo per ciastel ${ }^{l}$ (=pri ciastel), denominazione locale di un sentiero abbandonato nei pressi della rocca di Monrupino (Repentabor). A detta dell'informatore interrogato dal Lonza detta strada, che si snoda in direzione di Vogliano (Voglje), ricalcherebbe addirittura una vecchia strada romana. A parte l'esagerazione, ci troviamo di fronte ad un ulteriore indizio dell'antichità della denominazione, relitto 
indubitabile (a causa di quel CA passato appunto a cja) dello scomparso strato neolatino friulaneggiante che interessava anche questo lembo di territorio carsico. L'espressione toponimica (letter. "presso il castello", con allusione alla rocca di Monrupino o ad un vicino castelliere) è stata ben presto slovenizzata in una forma prettamente dialettale (e questa è un'altra garanzia della sua autenticita).

4. triest. Colòncovez:

Scendiamo per un momento dal ciglione carsico, spingendoci verso la città di Trieste, alla periferia della quale si trova la località di Colóncovez (ortografato anche Kolonkovez, o alla slovena, Kolonkovec), sita alla spalle dei Cimiteri, all'incirca tra Santa Maria Maddalena Inferiore e Monte Castiglione. Variante, un po' meno diffusa, del toponimo: Colòncavez (anche kolonkavec). Dato l'aspetto decisamente slavizzante del nome, si era tentato, nel passato, di sostituirlo con le dizioni burocratiche di costaIunga e con Piani (o Poggi) Sant'Anna. Ma I'innovazione non ha attecchito ed il popolo, esprimendosi in dialetto, continua ad usare appunto coloncovez e forme affini.

Il toponimo ha poca storia, tuttavia abbastanza significativa. Ia forma più anticamente attestata suona caloncovez e compare nell'Ortsreperterium del 1885 ( $\mathrm{p}$. 2): Caloncovez in A.

Scocchi "P.Or." (1938) p. 124 e in G. Pinguentini, Nuovo dizionario del dialetto triestino (Bologna 1969) p. 78, nonche ne "Il Piccolo" 29-3-1973 (parole di E. Marini: "1' esotico nome indigeno, che per la verita ricorre solo in qualche carta austriaca di un secolo fa..."). Ma già l'Ortsrepertorium del 1894 (p. 4) riporta la dizione più moderna kolonkavec (cfr. anche Jurišić, Topon, zapadne Istre c. 2 p. 57 e più recentemente Tržaško ozem1je F 7).

Sulla base di questa forma più antica e di altre considerazioni che riferiremo, l'etimo di questo toponimo, un unicum in tutta la regione ed aree linguistiche adiacenti (Friuli, Goriziano, Istria ecc.) - tant'è vero che il Bezlaj stesso, non essendo a conoscenza di certi "Realien" non riesce a darne una spiegazione, pur intuendo al fondo di esso un radicale "non slavo" (2) 
- può definirsi assolutamente sicuro: Colòncovez è un derivato di un lat. CANŌNICUS dissimilatosi in COLŌNICUS, forma che sta alla base del topon. veneto Calonega e, soprattutto, delle forme terg. cialònic (Dial. Mainati pp. 40, 73, 76 Do.), del friul. cialuni (N. Pir.), dell'albon. (dei "bilingui", Rosamani s. v.) calònic, nonché del triest. mod, calònego (Doria-Noliani GDDT s. v.). Ad ogni modo, a differenza di quanto succede per per Ciastel, il nostro Coloncovez presuppone una forma neolatina con ka- non palatalizzato, più prossima agli esiti venezianeggianti che non a quelli friulani dell'area in questione. E questo è certo un indizio di seriorità dell'accatto. Nondimeno il toponimo ha subito una slavizzazione assai profonda, in virtù dell'aggiunta dei sufissi -ov-ec- e della sincope della vocale atona $i$. La ragione del nascere di una simile denominazione, con base CANŌNICUS la ricaviamo da quanto ci riferisce il Marini (art. c.): nella località predetta esisteva nel secolo scorso un"osteria "Al canonico" al pianterreno di una casa un tempo proprieta di un vecchio canonico. Data questa circostanza non c'è affatto ragione di dubitare dell'esattezza dell'etimo proposto e nemmeno di aderire alla spiegazione di C. Desinan ("Metodi \& Ricerche". N. S. 2, 1983, p. 72), il quale vedrebbe nel toponimo un radicale slavo significante "scroscio". oppure il ben noto sost. kolo significante "cerchio, rotondità".

5. Žavlje

Premetto innanzitutto che intendo trattare qui di žavlje, un "Flurnamen" in quel di San Pelagio (Šempolaj), cosi come viene attestato in Tržaško ozemlje (C 2). Nonpertanto balza subito evidente l'omofonia $d i$ esso con il ben noto žavlje (ital. zàule), denominazione di. un centro abitato, un tempo a carattere prevalentemente rurale, e delle sue saline, ai confini tra il territorio di Trieste e di Muggia (Milje), di cui mi sono occupato a lungo anni fa (BDVI 1, 1972, pp. 43-47). Omofonia che è, anche, omonimia, trattandosi in ambedue $i$ casi di una stessa denominazione, un friulaneggiante zàule o zàole, letteralmente "piccola cesta di vimini" (cfr. triest. zaja, friul. zae "benna"), con allusione a certi avvallamenti del terreno, quasi una 
serie di piccole conche, facilmente individuabili sia nel paesaggio ondulato che circonda la piana di Zaule, sia nella landa carsica nei pressi, appunto, di San Pelagio. Anche per il Carso abbiamo a che fare, dunque, con un vecchio friulanismo, riconoscibile abbastanza agevolmente per il CA (si parte da un lat. JACA "giaciglio", poi "benna" ecc.), passato in posizione intervocalica a -ja-, con un $j$ (come quello di altra origine, cfr. triest. famea, meo "famiglia", "meglio") successivamente scomparso.

Colgo qui l'occasione per fornire ulteriori ragguagli su triest. zaule. La testimonianza più antica resta quella del 1236, ma si confronti anche un "in contrata Zaulis" dell'a. 1348 (D. Bloise "Arch. Tr." 40, 1980, p. 40) e a. 1354 (Camerario J. Gremon, ed. Persi-Cocevar "Arch. Tr." 42, 1982, p. 68). Notevoli anche alcune varianti, che allora non conoscevo, come mugg. moderno e triest. "negron" sàule (A. Branica "Robe Cusi" 1971 f. II p. 2 bis, G. Lipizzer Mondo Macaco, Trieste 1923, p. 36), il quale sta alla base anche di un "Saule" di una pianta di Trieste del 1798 redatta in lingua tedesca ("Salinen Von Sau1e" e "bey Saule" [cartiglio]), nonché zaole a. 1620 (M. Bertoša ACRSR 6, 1975-76, p. 151) e a. 1880 ("La Provincia dell'.Istria" 15 marzo, cfr. Apih-Colli Catalogo analitico della stampa periodica istriana I, Trieste 1983, p. 266) e zavole "La zenziva del Mare verso Zavole" a. 1594, in un discorso di F. Strassoldo, Seri-Ivanissevich San Vito, Trieste 1980, p. 85), zaula ("Contrada Zaula" a. 1583, P. Kandler Confini cit. p. 12) e zaulo ("Caleidoscopio (Trieste)" IV, 1845, p. 8). Nessuna di queste forme è in grado di smentire l'etimo da me proposto fin dal 1972, semmai, piuttosto, sarà da sottolineare che l'espressione del 1620 "valle detta Zaule" (M. Bertoša ACRSR cit. p. 142) potrebbe proporre un'equazione lessicale "zaule - vallecola" assai utile per $i$ nostri fini. Senza fondamento, evidentemente, l'etimo da ted. Zolle "dogana", riproposto di quando in quando da qualche studioso di cose locali (es. N.N. in "Istria Romantica", Trieste 1977, p. 13, cfr. ora A. Ancona in "Borgolauro" V, 5, 1984, p. 26) e poco convincente anche il za-ule "dietro 1'olivo" (slov. ant. ule, ulje "olivo") suggeritomi per lettera (maggio 1981) dall'informatore A. Švagelj (slov. za non puó essere confuso né 
scambiato con ža-: $\check{z}$ e z sono due fonemi del tutto diversi, aventi ciascuno una storia e una distribuzione ben definite).

1 Un cenno assai rapido su PER CIASTEL da parte dello scrivente in una relazione tenuta in occasione del Convegno della societa Italiana di Glottologia a Pescara, cfr. "Per la storia e la classificazione dei dialetti italiani" (Pisa 1980), p. 46

2 Il Bezlaj, 1..c., difatti propone, ma solo con molta esitazione, una base lat. ANCULUS (cogn.) o CALLANCULUS (dimin. di CALLIS).

3 Cfr. di sfuggita M. Doria, RID V-VI (I98I-82) E. 1 p. 154.

$\begin{array}{lllllllll}A & D & D & E & N & D & U & M\end{array}$

a Monte Coste: slov. kuósten "castagno" risulta, ora, attestato proprio per sgonico, cfr. Atlante storico Linguistico Etnografico Friulano, ed. G. B. Pellegrini, vol. V, Udine (1984) Carta N. 678. Cfr. anche il microtoponimo Kostanj lungo la strada Samatorza-Sales, Tržaško ozemlje cit. $D 2$.

a Coloncovez: Il tipo toponomastico veneto Calonega compare attestato anche per l'Istria: cfr. Calonoga a. 1538, nell'entroterra orserese, (D. Klen Fratrija, Rijeka 1969, p. 76 e n. 206).

Elenco di abbreviazioni

$A C R S R$

$A S P P$

Arch. tr.

$B D V I$

Bezlaj ESSJ

Dial. Mainati

Doria-Noliani $G D D T$

N. Pir.
Atti del Centro di Ricerche storiche di Rovigno Atti della Societa per la Preistoria e protostoria delle regioni Friuli-Venezia Giulia.

Archeografo. Triestino

Bollettino del Centro per lo studio dei dialetti veneti dell'Istria

F. Bezlaj, Etimološki slovar slovenskega jezika, Ljubljana 1980 ss.

G. Mainati, I dialoghi piacevoli in dialetto vernacolo triestino, ed. critica a cura di $M$. Doria, Trieste 1972 .

M. Doria, Grande dizionario del dialetto triestino... con la collaborazione di Cl. Noliani (a puntate sul "Meridiano di Trieste", genn. 1984 ss.).

A. G. Pirona-E. Carletti-G. B. Corgnali, Il Nuovo Pirona, vocabolario friulano, Udine 1935. 
$R D I$

Rosamani

$S L F$

Tržaško ozemlje

Wolf-Pleteršnik

zudini-Dorsi

Rivista di Dialettologia Italiana

E. Rosamani, Vocabolario giuliano, Bologna 1958. Studi Linguistici Friulani

Tržaško ozemlje. Seznam imen (a cura di un'équipe di studiosi), Ljubljana-Trst 1978

A. A. Wolf-M. Pleters̆nik, Slovensko-Nemški slovar, 1896 .

D. Dorsi-P. Zudini Dizionario del dialetto muglisano, Udine 1981

\section{Povzetek}

\section{PABERKOVANJA PO KRASKIH KRAJEVNIH IMENIH}

Avtor pretresa veljavnost dozdaj predlaganih etimoloških razlag za nekatera kraska krajevna imena. Vidi slovenski vir za Monte Coste (slov. kostanj), za Percedol (slov. pereć, deležnik glagola pereti, 'prhneti", 'gniti'); ugotavlja pa furlanski vir za per ciastel in sicer zaradi glasovne podobe, ki jo kaže lat. CASTELLUM (CA, KA $v$ ča, razločevalna črta furlanske fonetikel; ugotavlja romanski vir za Coloncovez, vendar ne furlanski, pać pa vexjetno beneski: razlog za ime naj bi bila neka krčma "Al canonico", ki je bila v l9. stol. v hiši nekega kanonika. Žavlje, ledinsko ime blizu Sempolaja, nic̆ drugače pa ne velja za žavlje, it. zaule med Trstom in Miljami, pa je iz furlanskega zaule 'majhna pletena košara'. 